\title{
Micro-RNA-21 rs1292037 A>G polymorphism can predict hepatocellular carcinoma prognosis (HCC), and plays a key role in cell proliferation and ischemia-reperfusion injury (IRI) in HCC cell model of IRI
}

\author{
Chaoyu Wu, MD, Gongen Tang, MD, Xiaoying Wang, MD, Jian Zhang, MD, Shuhua Chen, MD, Cuihong Lu, MD, \\ Dongqin Zhang, MD, Yun Li, MD.
}

\begin{abstract}

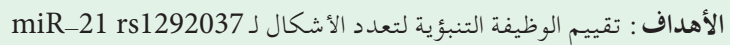
A<G . (HCC)

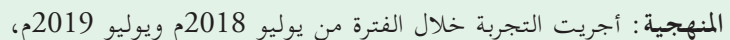

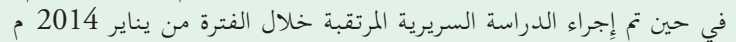

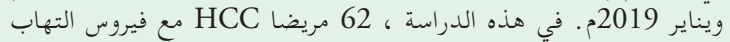

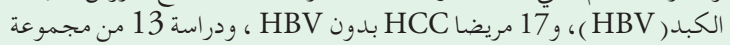

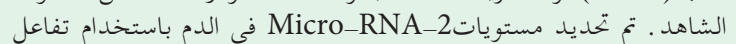

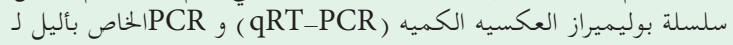

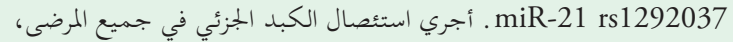

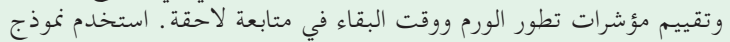

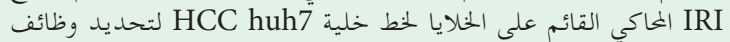

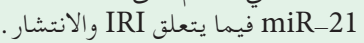

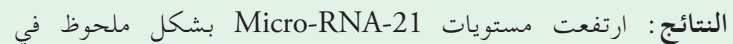

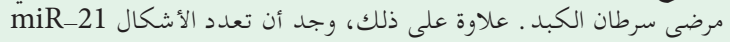

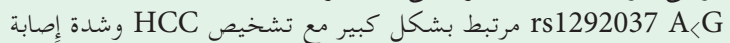

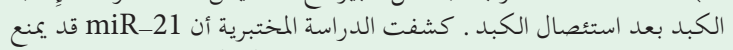

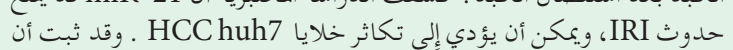

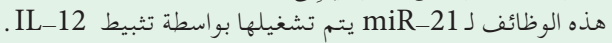

الخلاصة : تعدد أشكال Micro-RNA-21 rs1292037 A>G يمكن أن يتنبأ بتطور IRI وتطور الورم من خلال تنظيم التحال
\end{abstract}

Objectives: To assess the predictive function of miR-21 rs1292037 A>G polymorphism in survival and ischemia-reperfusion injury (IRI) in hepatocellular carcinoma (HCC) patients.

Methods: The experiment was carried out between July 2018 and July 2019, whereas the prospective clinical study was carried out between January 2014 and January 2019. In this study, 62 HCC patients with hepatitis B virus (HBV), 17 HCC patients without $\mathrm{HBV}$, and 13 healthy controls were investigated. Micro-RNA-21 levels in the blood were identified using quantitative reverse-transcription polymerase chain reaction (qRT-PCR) and allele specific PCR for
miR-21 rs1292037.Partialhepatectomywasperformed in all patients, and the tumor development indicators and survival time were evaluated in a subsequent follow-up. Cell-based simulated IRI model of HCC huh7 cell line was used to determine the functions of miR-21 with respect to IRI and proliferation.

Results: Micro-RNA-21 levels were significantly increased in HCC patients. Furthermore, an miR$21 \mathrm{rs} 1292037 \mathrm{~A}>\mathrm{G}$ polymorphism was found to be significantly associated with HCC prognosis and severity of liver injury after hepatectomy. In vitro study revealed that miR-21 might prevent IRI from occurring, and can induce proliferation in HCC huh7 cells. These functions of miR-21 were demonstrated to be triggered by IL-12A inhibition.

Conclusion: Micro-RNA-21 rs1292037 A>G polymorphism can predict IRI and tumor progression by regulating the miR-21/IL-12A.axis.

Keywords: hepatocellular carcinoma, polymorphism, ischemia-reperfusion injury, microRNA, proliferation

Saudi Med J 2020; Vol. 41 (4): 383-392 doi: 10.15537/smj.2020.4.24994

From the Department of Infectious Diseases, Linyi Central Hospital, Linyi, China.

Received 5th September 2019. Accepted 3rd February 2020.

Address correspondence and reprint request to: Dr. Yun Li, Department of Infectious Diseases, Linyi Central Hospital, Linyi, China.E-mail: liyunlyzxyy@sina.com

ORCHID ID: http://www.orcid.org/ 0000-0002-5921-8675 
$\mathrm{T}$ The incidence and mortality rates associated with hepatocellular carcinoma (HCC) rank among the top 5 cancer-related mortality and incidence rates in the world, and the second in China. ${ }^{1}$ Techniques such as local ablation have been developed to treat HCC, but despite these advances, hepatectomy is still the most effective treatment. However, the survival rates of HCC remain very low mainly because of its high recurrence. ${ }^{2}$

Hepatic ischemia-reperfusion injury (IRI) has recently been reported to be a major factor in accelerated tumor growth. Ischemia-reperfusion injury induces the release of growth factors and proinflammatory factors, causing liver microenvironment changes, thus, promotes tumor spread and metastasis. Some studies have revealed that the expression disorder of certain indicators related to IRI can predict the prognosis of HCC patients. ${ }^{3}$

A new class of short-chain noncoding ribonucleic acids (RNAs) called microRNAs (miRNAs) can inhibit the post-transcriptional expression of genes, and thus can regulate a variety of pathophysiological responses. ${ }^{4}$ The abnormal expression of miRNAs caused by external stimulation or self-factors can change tumor-related signaling pathways, hence regulating many different tumor development processes, including HCC. In addition, miRNA expression has been shown to be useful in understanding hepatocarcinogenesis, its diagnosis, and prognosis. ${ }^{4,5}$

Mutations in the promoter regions of certain miRNAs usually cause changes in their expression. ${ }^{6.7}$ In addition, single nucleotide polymorphisms (SNPs) located in some miRNAs may be closely related to their binding ability to target mRNAs. ${ }^{8}$ Therefore, these SNPs might affect the individualized differences in their expression or functions; therefore, it predict the prognosis of certain diseases. Our recent study demonstrated that miR-21, which has been reported to be involved in the proliferation, migration, and invasion cancer cells, was significantly upregulated in HCC patients after liver resection. ${ }^{9}{ }^{, 10}$ However, whether the miR-21 rs 1292037 $A>G$ polymorphism is related to the expression of miR-21, which cell functions are regulated by the miR-21 rs1292037 $A>G$ polymorphism, and whether the miR-21 rs1292037 $A>G$ polymorphism is related to the prognosis of HCC are all still unknown.

Disclosure. Authors have no conflict of interests, and the work was not supported or funded by any drug company.
The objective of this study is to further investigate whether miR-21 and its gene polymorphisms regulate the occurrence and development of HCC and to determine whether miR-21 inhibits the development of cancer by protecting against IRI.

Methods. This study was carried out in Linyi Central Hospital, Linyi, China. The experimental study was carried out between July 2018 and July 2019, whereas the prospective clinical part was carried out between January 2014 and January 2019. We used the PubMed Modular of National Center for Biotechnology Information (NCBI) website to search for prior related publications.

Inclusion criteria of the case group are described as follows: HCC patients with or without hepatitis B vires (HBV) infection who need partial hepatectomy without contraindication. Patients younger than 18 years or older than 80 years, patients with a history of additional ablation or with cardiovascular and cerebrovascular complications were excluded from this study. In total, 62 HCC patients with HBV, 17 HCC patients without $\mathrm{HBV}$, who underwent liver resection surgery in Linyi Central Hospital, Linyi, China were included in this study, together with 13 healthy volunteers as controls (HCs). Their bodily functions, sizes and locations of their lesions, their blood biochemistry, and liver functions were determined and recorded, in accordance to our previously reported methods. ${ }^{11}$ Blood samples before and after surgery and of healthy patients were collected to monitor liver function. Hepatocellular carcinoma tissue samples were also obtained. The study performed was according to the principles of Helsinki Declaration. Informed consent was obtained from all patients before participation. Ethics Committee of the Linyi central hospital approve this study.

Ribonucleic acid extraction. Ficoll density gradient centrifugation (Amersham Pharmacia Biotech, Tokyo, Japan) was used to separate leukocytes from the blood samples of HCC patients before surgery and of HCs. Then, Qiagen miRNeasy Mini Kit (Qiagen, Hilden, Germany) was used to extract the total RNA from blood leukocytes and tissues. Ribonucleic acid was kept at $-80^{\circ} \mathrm{C}$ before analysis.

Quantitative reverse-transcription polymerase chain reaction ( $q R T-P C R)$. The $\mathrm{QRT}-\mathrm{PCR}$ detection reagents used for miRNA and mRNA were TaqMan ${ }^{\text {TM }}$ MicroRNA Assay kit and PrimeScript RT-PCR kit, which were purchased from Thermo Fisher (item no. 4440888, Waltham, USA) and Takara (item no. RR037A, Shiga, Japan), respectively. iQ5 real time fluorescence quantitative PCR instrument was used 
for qRT-PCR analyses (Bio-Rad, Hercules, CA, USA). The parameters of the reverse transcription and PCR reactions were set according to our previous description. ${ }^{11}$ The primer sequences for miR-21 were forward, 5'-GCCGCTAGCTTATCAGACTGATGT-3', and reverse, 5'-GTGCAGGGTCCGAGGT-3'. The primer sequences for U6 were forward, 5'-GCTTCGGCAGCACATATACTAAAAT-3', and reverse, 5'-CGCTTCACGAATTTGCGTGTCAT-3'. The primer sequences for IL-12A were forward, 5'-CCTTGCACTTCTGAAGAGATTGA-3', and reverse, 5'-ACAGGGCCATCATAAAAGAGGT-3'. The primer sequences for $\beta$-actin were forward, 5'-TCCTCTCCCAAGTCCACACAGG-3', and reverse, 5'-GGGCACGAAGGCTCATCATTC-3'. Relative expression is calculated by comparing the threshold period (CT) between targets and internal references.

Genotyping. We extracted genomic deoxyribonucleic acid from patients' blood samples and the miR-21 rs1292037 polymorphisms were determined by ligase detection reaction-PCR according to the previous report. $^{12}$

Cell culture and simulation of IRI. Primary cultures from the HCC tissues were prepared. Fresh HCC tissue samples were washed with phosphate-buffered saline, cut into pieces, and incubated in trypsin solution. Then, it was slowly stirred and digested in a $37^{\circ} \mathrm{C}$ water bath for 15 to 20 minutes, and the product was used for biopsy. Subsequently, it was filtered using a $70 \mu \mathrm{m}$ filter paper to remove large pieces of undigested lumps. After centrifugation, the precipitates were mixed, and the recovered cells were suspended in DMEM/Ham's F12 nutrient mixture (F12). B27, basic fibroblast growth factor, $(20 \mathrm{mg} / \mathrm{ml})$ and epidermal growth factor $(20 \mathrm{mg} / \mathrm{ml})$ were added to the medium.

Human hepatoma cell line, huh7, was identified by Short Tandem Repeat (STR) by LMAI Bio (LMAI Bio, Shanghai, China), and conventionally cultivated and passed on in our laboratory. Ischemia-reperfusion injury models were established as previously described. ${ }^{13,14}$ The cells were briefly treated with a ischemic solution to simulate an ischemic environment, and were exposed to $1 \% \mathrm{O}_{2}$ (EXP group) 24 hours after they were inoculated into the culture plate. Two hours after the ischemia treatment (ischemia period), normoxic Krebs-Henseleit solution was used to start the reperfusion period. ${ }^{13}$ After 180 minutes of conventional cultivation, the cells were harvested (reperfusion period). The cells not treated with ischemia solution and cultured under normoxic conditions were used as controls (CTL group), which were inoculated and harvested at the same time as the EXP group.

Cellular transfection. Shanghai GenePharma Co., Ltd. (Shanghai, China) provided oligonucleotides for overexpression (mimics), inhibition (inhibitor), and control (miR-control) of miR-21. The cell transfection reagent was Lipofectamine ${ }^{\mathrm{TM}}$ 2000, which was purchased from Item No. 11668027, Waltham, USA. Following the manufacturer's instructions, the cells were transfected with these oligonucleotides at $50 \mathrm{~nm}$ to simulate the cell environment of miR-21 overexpression or inhibition.

Statistical analysis. Statistical Package for Social Sciences for Windows, version 19 (IBM Corp, Armonk, NY, USA) was used to analyze the experimental data. Each experiment was repeated 3 times, and the results were expressed as mean \pm standard deviation. The differences between the 2 groups were statistically analyzed using 2-tailed t-test. A $p$-value less than 0.05 was considered to have a statistical difference. Chi-square test or Fisher exact test was used to analyze the relationship between miR-21 rs1292037 gene polymorphism and clinical indicators.

Results. The expression of miR-21 in HCCpatientswas significantly up-regulated. The detailed demographics of the subjects were described. No differences in gender, age, smoking, and alcohol consumption were found among the 3 groups. The expression of miR-21 in HCC patients was significantly up-regulated compared with that of HCs, whereas HBV infection does not affect the expression of miR-21 (Figure 1A). Additionally, miR-21 expression in leukocytes was consistent with that in HCC tissues (Figure 1B), and the level of miR-21 in HCC tissues was significantly higher than that in adjacent tissues (Figure 1C). The HBV infection group exhibited enhanced miR-21 levels in HCC tissues, as compared to that of HBV-negative patients (Figure 1D). Based on the receiver operating characteristic (ROC) curves, we found that miR-21 levels in blood leukocytes discriminated HCC patients from HCs. The area under the curve (AUC) of miR-21 was 0.797 (95\% CI $=0.694-0.901)$. For the control group, the AUC of serum alpha fetoprotein (AFP) was 0.723 $(95 \% \mathrm{CI}=0.584-0.861)$, as shown in Figure 2. We then divided HCC patients into high (33 cases) and low (46 cases) leukocyte miR-21 level groups, according to the optimal cut-off value (relative miRNA value $=1.51$ ) . As shown in Table 1, leukocyte miR-21 levels correlate with large tumor volume, late stage, and lymphatic metastasis. From the Kaplan-Meier analysis, we found that low levels of miR-21 were associated with higher 
A

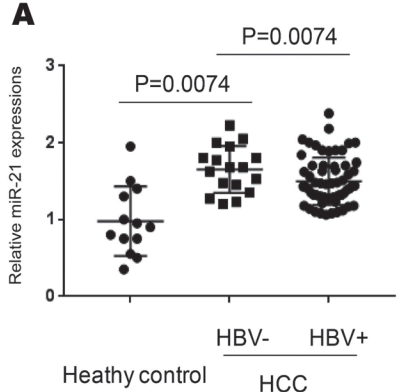

B

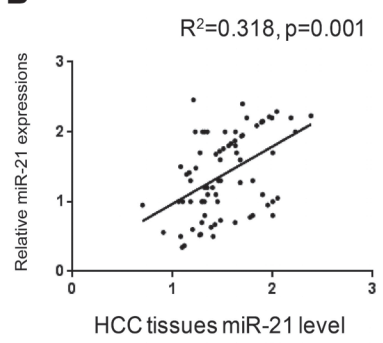

C

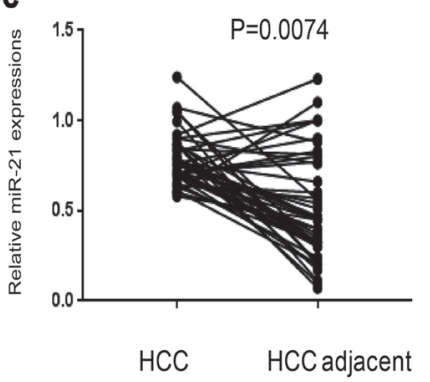

D $\quad \mathrm{P}=0.0004$

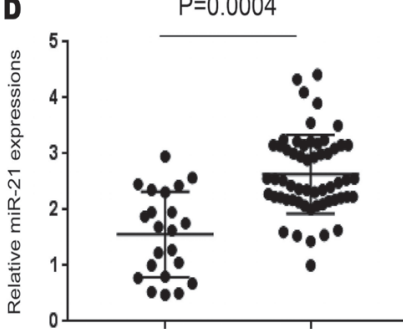

HBV-

$\mathrm{HBV}+$

Figure 1 - Micro-RNA-21 expression increased in hepatocellular carcinoma (HCC) patients. A) Quantitative polymerase chain reaction results comparing miR-21 levels in blood leukocyte among hepatitis B virus negative (HBV-) HCC patients, HBV positive HCC patients and healthy controls. Significant differences were calculated by non paired student-t test. B) Quantitative polymerase chain reaction results demonstrating the relationship between blood leukocyte miR-21 levels and HCC tissues miR-21 levels. C) Quantitative polymerase chain reaction results comparing the expression levels of miR-21 in HCC tissues and matched tumor-adjacent tissues. Significant differences were calculated by paired student-t test D) Quantitative polymerase chain reaction results comparing miR-21 levels between HBV negative and HBV positive HCC tissues. Significant differences were calculated by non paired student-t test.

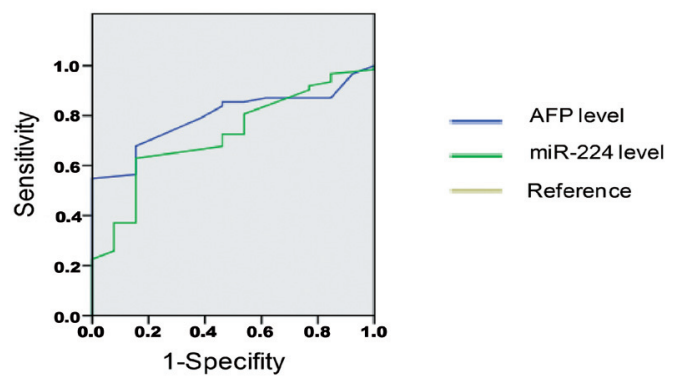

Figure 2 - The receiver operating characteristic curve for blood leukocyte miR-21 level and serum AFP level in relation to the hepatocellular carcinoma patients.

Table 1 - Micro-RNA-21 level in hepatocellular carcinoma patients.

\begin{tabular}{lcccc}
\hline & $\begin{array}{c}\text { Low } \\
(\mathbf{n}=46)\end{array}$ & $\begin{array}{c}\text { High } \\
(\mathrm{n}=33)\end{array}$ & $P$-value & OR $(95 \% \mathrm{CI})$ \\
\hline $\begin{array}{l}\text { Age } \\
>55\end{array}$ & 26 & 18 & 0.862 & $1.083(0.441-2.664)$ \\
$\quad<55$ & 20 & 15 & & \\
$\begin{array}{l}\text { Gender } \\
\quad \text { Male }\end{array}$ & 34 & 23 & 0.680 & $1.232(0.457-3.322)$ \\
$\quad$ Female & 12 & 10 & & \\
$\begin{array}{l}\text { HBV infection } \\
\text { Negative }\end{array}$ & 14 & 3 & 0.023 & $4.375(1.142-16.754)$ \\
$\quad$ Positive & 32 & 30 & & \\
Stage & & & & \\
$\quad$ I or II & 28 & 8 & 0.001 & $4.861(1.802-13.111)$ \\
$\quad$ III or IV & 18 & 25 & & \\
$\begin{array}{l}\text { Lymphatic } \\
\text { metastasis }\end{array}$ & & & & \\
$\quad$ No & 27 & 12 & 0.050 & $2.487(0.990-6.244)$ \\
$\quad$ Yes & 19 & 21 & & \\
Tumor size & & & & \\
$\quad 55$ & 31 & 16 & 0.091 & $2.196(0.875-5.509)$ \\
$\quad 55$ & 15 & 17 & & \\
\hline
\end{tabular}

HBV: hepatitis B virus, OR: odd ration, CI: confidence interval
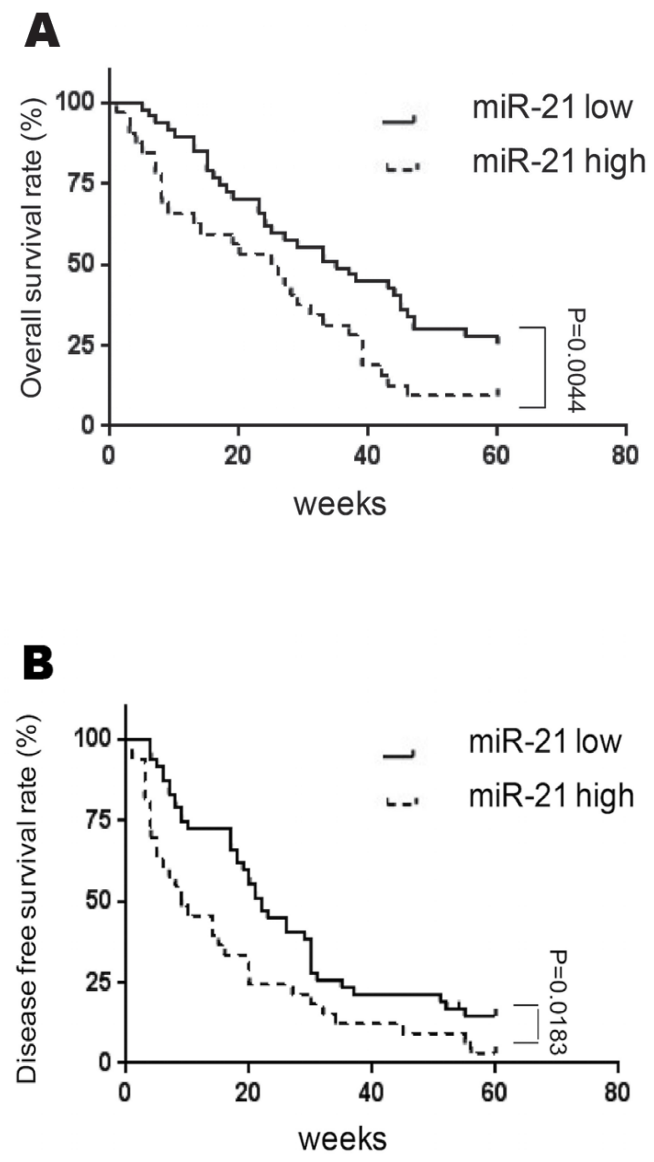

Figure 3 - Kaplan-Meier curves for time to A) overall survival and B) disease-free survival of patients according to different miR-21 level. 
overall survival (OS) prognosis (Figures 3A-B) and longer disease-free survival (DFS), as shown in Figures 3A-B.

MiR-21 rs 1292037 polymorphism of HCC patients was correlated with miR-21 expression, clinical characteristics, and prognosis. We subsequently explored the association of rs1292037 polymorphism in HCC patients with clinical characteristics and prognosis. There were 22 patients with the AA genotype at miR-21 rs1292037, whereas 57 patients carried the AG/GG genotype. First, we found that levels of miR-21 in leukocytes has significantly decreased in patients with the AA genotype, as compared with those in the AG/GG group (Figure 4). As shown in Table 2, those with the AA genotype exhibited less susceptibility to $\mathrm{HBV}$, as compared with those with the AG/GG genotype. Additionally, those with the AA genotype possessed less late stage (III or IV) diseases, larger tumor size (greater than $5 \mathrm{~cm}$ ), and lymphatic metastasis than AG/GG cases, but the difference in their $p$-values compared with each other is not less than 0.05. KaplanMeier analysis indicated that the AA genotype showed more accurate $\mathrm{OS}$ prognosis (Figure 5A) and longer DFS, as seen in Figure 5B. Next, we tested whether the combination of miR-21 rs 1292037 polymorphism and $\mathrm{HBV}$ positive results is a more accurate predictor of $\mathrm{OS}$ and DFS. Patients with the $\mathrm{AA}+\mathrm{HBV}^{\text {negative }}$ phenotype showed the highest OS and DFS rates, and significant differences in the survival rates between $\mathrm{AA}+\mathrm{HBV}^{\text {negative }}$ and $\mathrm{AA}+\mathrm{HB}^{\text {positive }}$ patients were found. However, the difference between $A G / G G+H B V$ negative and $\mathrm{AG} / \mathrm{GG}+\mathrm{HBV}^{\text {positive }}$ types was found to be insignificant (Figure 6).

Micro-RNA-21 rs1292037 polymorphism was related to hepatic IRI of HCC patients after hepatectom. Some groups' findings suggest that the indicators of IRI severity after hepatectomy can function as potent markers for HCC prognosis because severe IRI at the early phase after hepatectomy or live transplant provides a good environment for tumor growth. ${ }^{3}$ Serum alanine aminotransferase (ALT) levels a day post-resection were significantly increased compared with baseline, gradually decreasing until seven days post-resection. Compared to AA cases, AG/GG genotype group had higher serum ALT levels a day post-resection. However, after 7 days, both ALT and aspirate aminotransferase (AST) values of group AG+GG were lower than those of the AA group, and were lowest in the AG+GG genotype group (Figure 7A). Similar results were also observed with AST (Figure 7B). However, total bilirubin test and DBIL, which were also potent indicators for IRI, did not correlate with miR-21 rs1292037 polymorphism (Figures 7C-D).
MiR-21 prevents HCC cell injury and promotes proliferation in an in vitro simulated IRI model. We next established an in vitro simulated IRI model using the human HCC cell line Huh7. The miR-21 expression increased in Huh7 cells during the ischemic and reperfusion periods, compared with that of the control group. Furthermore, compared with the ischemic period, miR-21 levels increased in the reperfusion period (Figure 8A). Previous reports demonstrated that receiver operating characteristics (ROS) pathway mediates the cell injury upon exposures to ischemia and

Table 2 - Genotype distributions of miR-21 rs1292037 polymorphism in hepatocellular carcinoma patients.

\begin{tabular}{lcccc}
\hline & $\begin{array}{c}\text { AA } \\
(\mathrm{n}=22)\end{array}$ & $\begin{array}{c}\text { AG/GG } \\
(\mathrm{n}=57)\end{array}$ & $P$-value & OR (95\% CI) \\
\hline $\begin{array}{l}\text { Age } \\
>55\end{array}$ & 13 & 31 & 0.706 & $1.211(0.447-3.283)$ \\
$\quad 555$ & 9 & 26 & & \\
$\begin{array}{l}\text { Gender } \\
\text { Male }\end{array}$ & 16 & 41 & 0.943 & $1.041(0.346-3.132)$ \\
$\quad$ Female & 6 & 16 & & \\
$\begin{array}{l}\text { HBVinfection } \\
\text { negative }\end{array}$ & 8 & 9 & 0.046 & $3.048(0.991-9.374)$ \\
$\quad$ Positive & 14 & 48 & & \\
Stage & & & & \\
I or II & 14 & 22 & 0.146 & $2.248(0.747-6.764)$ \\
$\quad$ III or IV & 8 & 35 & & \\
$\begin{array}{l}\text { Lymphatic } \\
\text { metastasis }\end{array}$ & & & & \\
$\quad$ No & 12 & 27 & 0.098 & $0.390(0.126-1.208)$ \\
$\quad$ Yes & 10 & 30 & & \\
Tumor size & & & & \\
$\leq 5$ & 14 & 33 & 0.071 & $0.37(0.124-1.107)$ \\
$>5$ & 8 & 24 & & \\
\hline
\end{tabular}

HBV: hepatitis B virus, OR: odd ration, CI: confidence interval

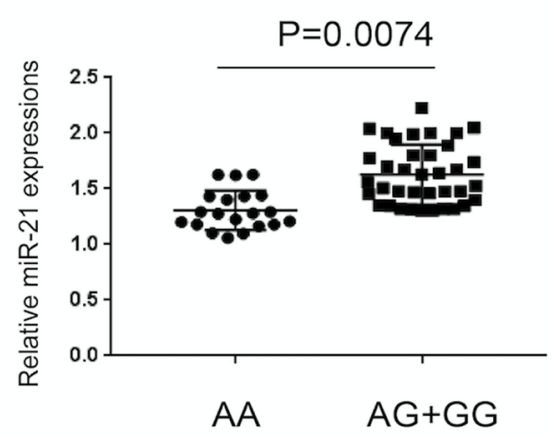

Figure 4 - Association of miR-21 rs1292037 polymorphism with the miR-21 level in blood leukocyte of the hepatocellular carcinoma patients. 

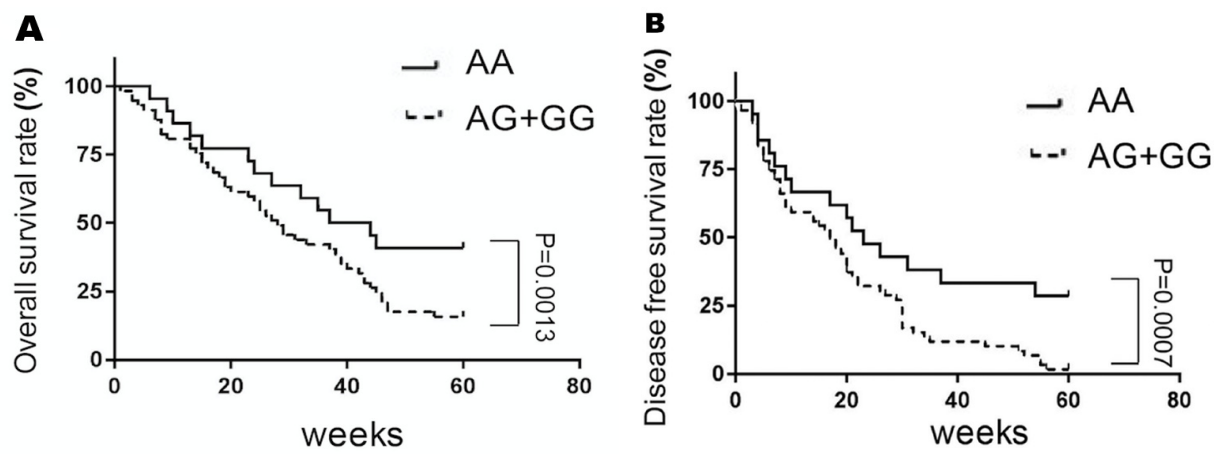

Figure 5 - Kaplan-Meier curves for time to A) overall survival and B) disease-free survival of patients according to different genetic polymorphism at genetic polymorphism at miR-21 rs1292037 SNP site.

A

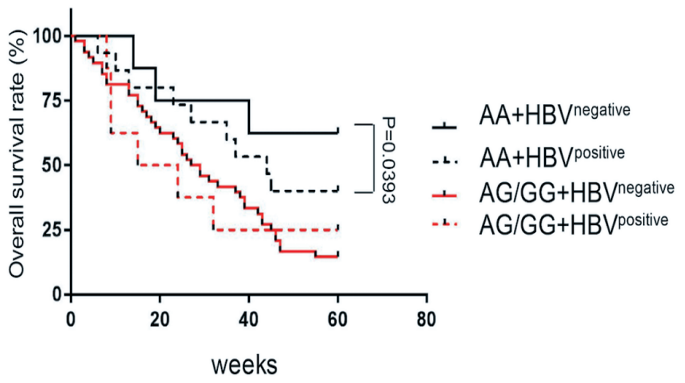

B

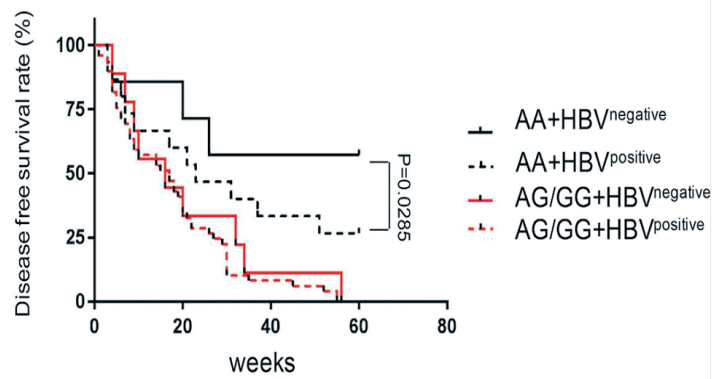

Figure 6 - Kaplan-Meier curves for time to A) overall survival and B) disease-free survival of patients according to combined miR-21 rs1292037 polymorphism and hepatitis B virus infection status.

A

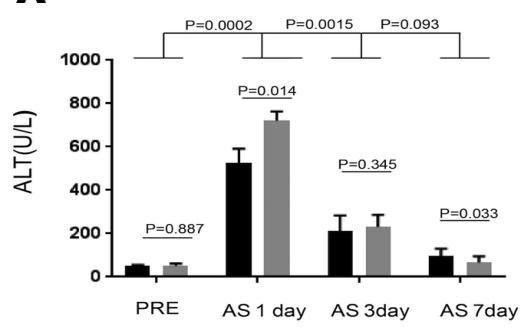

C

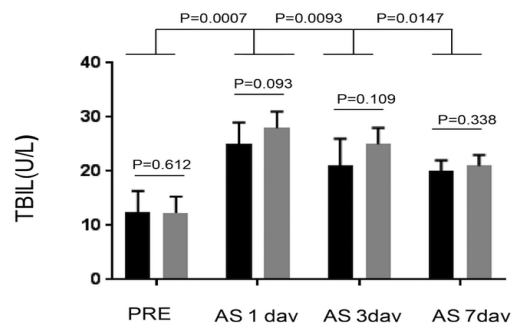

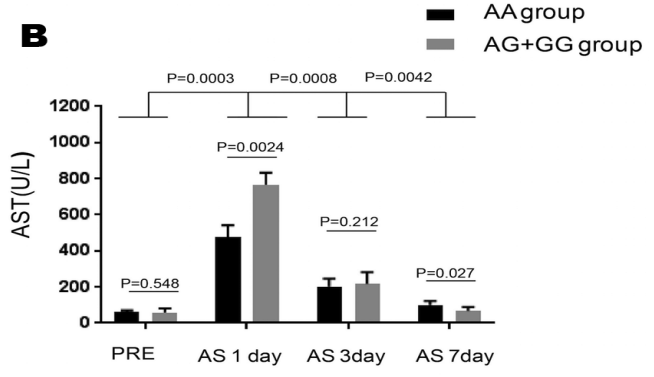

D

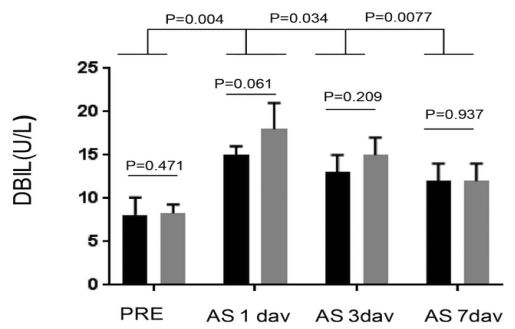

Figure 7 - Serum A) Alanine aminotransferase, B) aspirate aminotransferase, C) total bilirubin test, and D) direct bilirubin levels before and after partial hepatectomy of hepatocellular carcinoma patients with different genetic polymorphism at miR-21 rs1292037 polymorphism single nucleotide polymorphisms site. AA: miR-21 rs1292037 genotype is AA; AG/GG: miR-21 rs1292037 genotype is AG or GG. PRE: before hepatectomy AS 1 day: 1 day after hepatectomy; AS 3 day: 3 day after hepatectomy; AS 7 day: 7 day after hepatectomy. 
reperfusion. In this study, Huh7 cells were transiently transfected with miR-21 overexpressing or inhibitor oligonucleotides, and then were exposed to an IRI environment. We found that ROS accumulated during the acute ischemic process and persisted through reperfusion in Huh7 cells, whereas pre-treatment with miR-21 mimics decreased ROS accumulation (Figure $8 \mathrm{~B}$ ), indicating that miR-21 could attenuate ischemia-reperfusion-induced injury. Additionally, the IRI environment up-regulated Huh7 cell proliferation, whereas miR-21 inhibitor reversed the regulation of IRI-induced proliferation (Figure 8C).

MiR-21 protects against HCC cell injury and promotes proliferation by targeting proinflammatory factors IL-12A. Previous studies revealed that inflammation plays an important role in promoting IRI. Our luciferase reporter vector-based experiments demonstrated that interleukin (IL)-12A was a viable target of miR-21 in vitro (Figures 9A-B). Furthermore, miR-21 mimics downregulated the IL-12A mRNA level
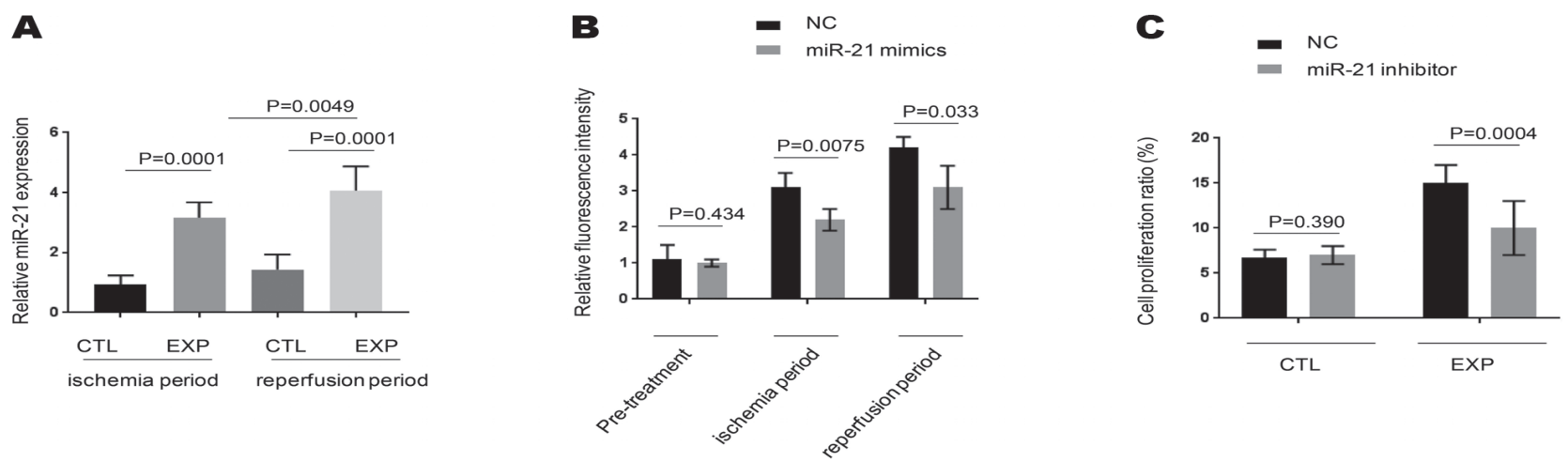

Figure 8 - Micro-RNA-21 protect hepatocellular carcinoma cell injure and promote proliferation. A) Huh7 cells were exposed to simulated IRI environment or not, and then quantitative polymerase chain reaction were used to determine the miR-21 levels in both ischemic period and reperfusion period; B) Huh7 cells were transfected with miR-21 mimincs or miR-21 NC, following with simulated IRI exposure, and then intracellular ROS levels were measured by a fluorometry assay using intracellular oxidation of DCFH-DA; C) Huh7 cells were transfected with miR-21 mimincs or not, following with simulated IRI exposure or not, and then the cell proliferation were determined.
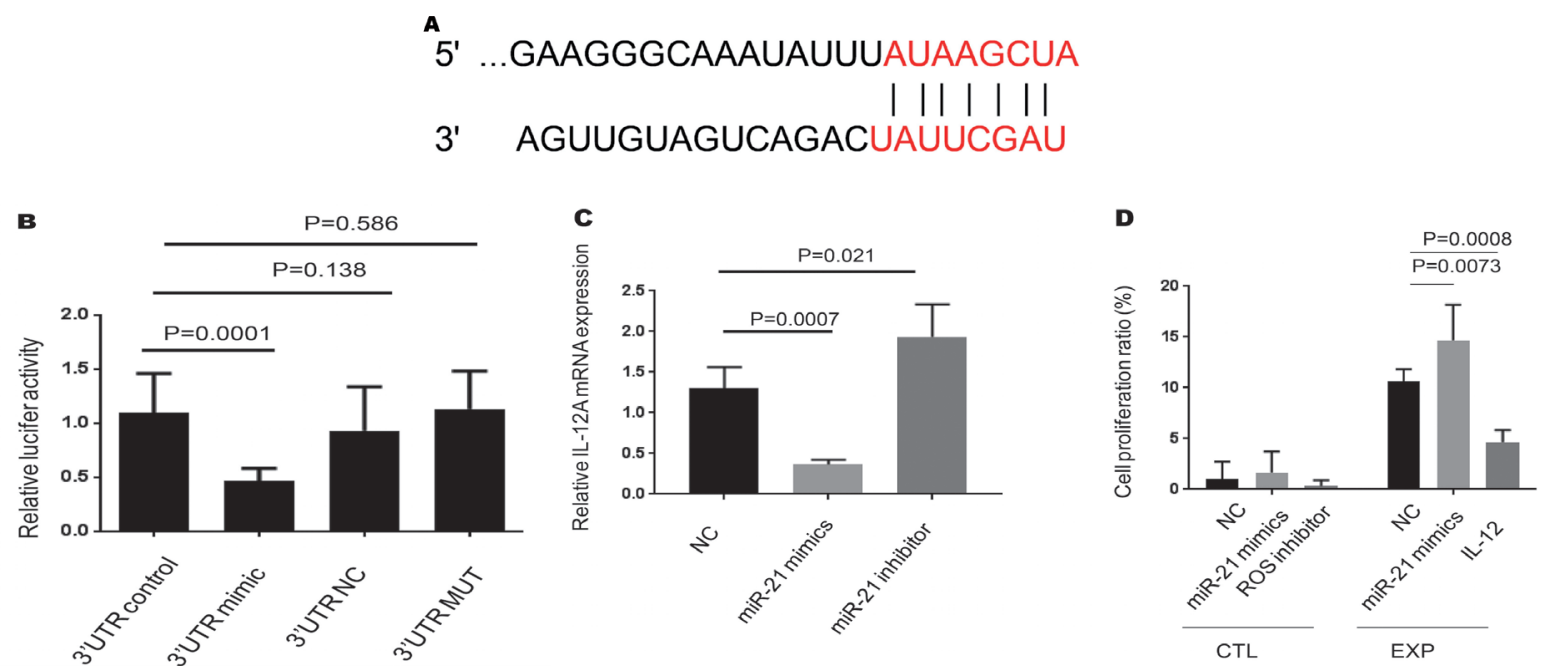

Figure 9 - Relative lucifer activity. A) The region of the human TNF $\alpha$ mRNA 3'UTR predicted to be targeted by miR-21. B) 293T cells were transiently co-transfected with luciferase reporter vectors for TNF $\alpha$, and either miR-21 mimics, inhibitor or negative control. Luciferase activities were normalized to the activity of Renilla luciferase. C) Huh7 cells were transfected with miR-21 mimincs, inhibitor or miR-21 NC, following with simulated ischemia-reperfusion injury (IRI) exposure, and then intracellular IL-12A mRNA expression were determined by qRT-PCR assay. D) Huh7 cells were transfected with miR-21 mimincs or miR-21 negative control, together with IL-12 treatment (10ng/mL) or not, following with simulated IRI exposure, and then the cell proliferation were determined 
in the simulated IRI model (Figure 9C). Huh7 cells were transiently transfected with miR-21 mimics, together with IL-12 treatment $(10 \mathrm{ng} / \mathrm{mL})$ or not, followed by simulated IRI exposure. We found that the regulatory functions of miR-21 in Huh7 cell proliferation were reversed by IL-12A treatment (Figure 9D).

MiR-21 rs1292037 polymorphism was associated with the miR-21 functions in HCC cells by regulating IL-12A expressions. We isolated and cultured HCC cells from 79 patients and found that miR-21 expression in cells with AA genotype was lower than that with AG/GG (Figure 10A). When these cells were exposed to an IRI environment, the AA group exhibited enhanced ROS accumulation and inhibited cellular proliferation, compared with AG/GG cases (Figures 10B-C). Furthermore, IL-12A expressions in both HCC cells and blood leukocytes were higher in AA group (Figures 10D-E). Collectively, the cell-based results and clinical findings demonstrated that miR-21 rs1292037 polymorphism functions on miR-21 expression of HCC cells, and therefore regulates IL-12A-mediated cell injury and proliferation.

Discussion. Different genetic characteristics are one of the important factors accounting for the differences in prognosis of patients with HCC; therefore, they could function as potential biomarkers. ${ }^{15}$ Single nucleotide polymorphisms of certain inflammatory cytokines, such as tumor necrosis factor alpha and IL-10, can regulate important carcinogenic signals and can affect the prognosis of HCC. ${ }^{16}$ Furthermore, genetic variation in miRNAs can alter their functions and that of their targets; hence, may be conducive to cancer progressions. ${ }^{17}$

Micro-RNA-21 has been reported to act as an inflammatory inhibitor, suppressing the expression of proinflammatory targets by binding to their 3'UTR. ${ }^{18-20}$
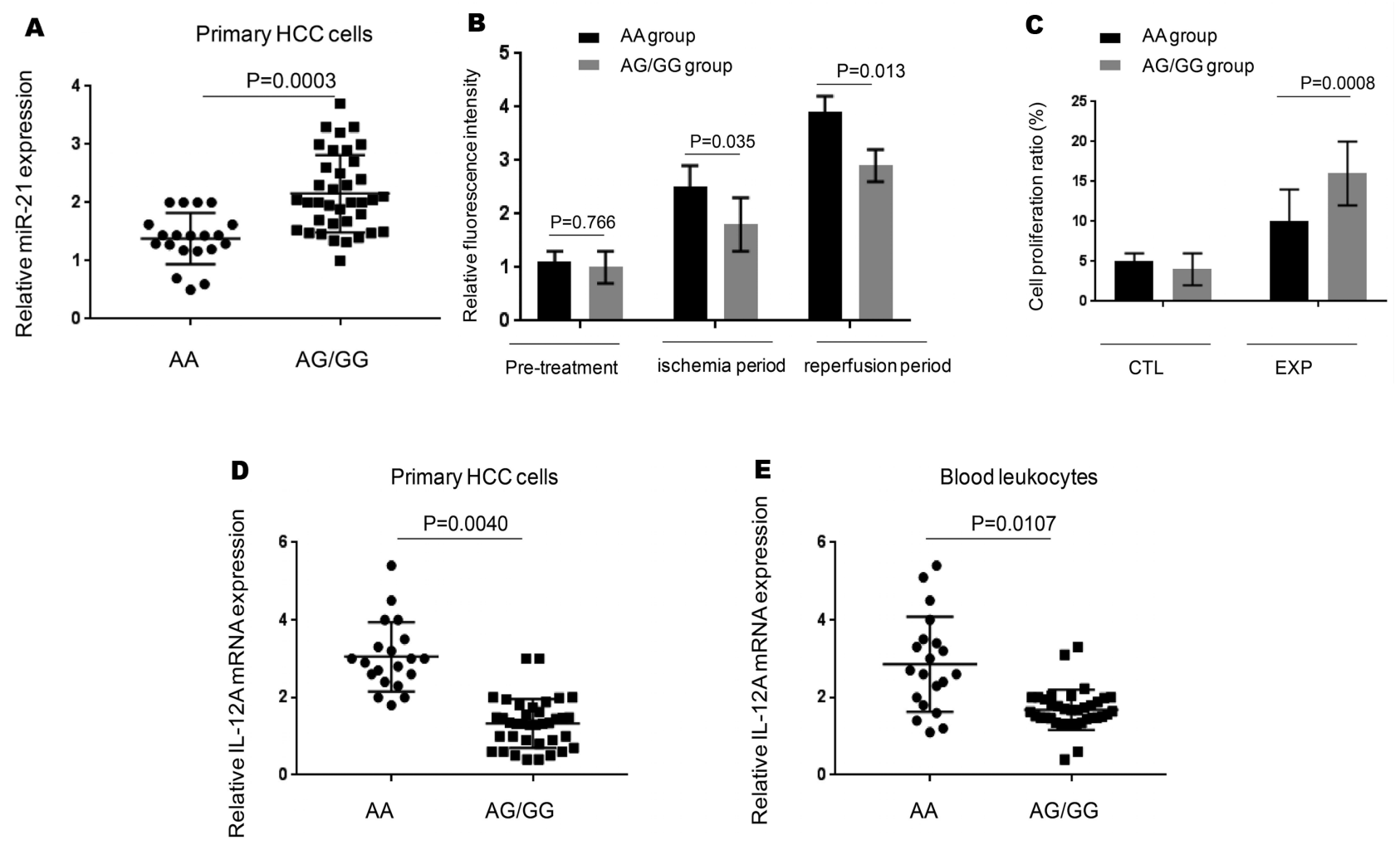

Figure 10 - MiR-21 rs1292037 polymorphism was associated with the miR-21 functions in hepatocellular carcinoma cell (HCC) cells by regulating IL-12A expressions. A) Primary HCC cells were isolated from 22 cases of rs 1292037 AA and 57 cases of AG/GG and were incubated for 24 h. The cells were harvest and the miR-21 expressions were assayed by quantitative reverse-transcript polymerase chain reaction (qRT-PCR). B) Cells in (A) were simulated IRI exposure, and then intracellular receiver operating characteristic levels were measured by a fluorometry assay using intracellular oxidation of DCFH-DA. C) Cells in (A) were simulated IRI exposure or not, and then the cell proliferation were determined. D) Cells in (A) were harvest and the IL-12A expressions were assayed by qRT-PCR. E) quantitative polymerase chain reaction results demonstrating the relationship between miR-21 rs1292037 polymorphism and IL-12A expressions in blood leukocyte of the HCC patients. 
Although substantial research has been conducted to investigate the functions of miR-21 in tumors, the results remain inconclusive. Similarly, studies on miR-21 expression patterns in HCC patients are also conflicting. Zhuang et $\mathrm{a}^{21}$ reported that miR-21 increased in HCC serum samples and can serve as a potential biomarker. Huang et $\mathrm{al}^{22}$ also found that increased expression of miR-21 could predict poor prognosis of HCC patients. In contrast, Yi et $\mathrm{al}^{23}$ did not find any association between miR-21 level and HCC prognosis. Our study found that higher miR-21 levels exist in blood leukocytes of HCC patients and HCC tissues than in non-HCC control patients. Subgroup analysis indicated that in patients with HCC, HBV infection can cause up-regulation of miR-21. Combined with previous evidence, these results suggest an association between miR-21 and HBV-related HCC pathogenesis.

The highlight of this study was finding out that miR-21 rs1292037 polymorphism in HCC patients is correlated with clinical indicators and prognosis. Increased OS and DFS rates were observed for patients carrying the rs1292037 AA phenotype. Moreover, the combined survival analysis of patients with the miR-21 rs1292037 polymorphism and HBV infection revealed that the $\mathrm{AA}+\mathrm{HBV}^{\text {negative }}$ phenotype predicted the highest OS and DFS rates. It revealed that miR-21 level and polymorphism, either separately or in combination, could be indicators of HBV-related HCC progressions and prognosis.

Notably, our results also indicated that some patients who carry miR-21 rs1292037 AG+GG phenotype had higher ALT and AST levels a day after hepatectomy. This may be attributed to larger and more aggressive tumors in $A G+G G$ patients, resulting in stronger IRI after surgery. However, interestingly, the recovery from this injury was faster in AG+GG patients one to 7 days after surgery, and this might be due to high miR-21 levels in $\mathrm{AG}+\mathrm{GG}$ cases, contributing to protection against IRI.

The polymorphisms of miRNAs can affect their expression or their binding to target genes, thus altering their biological functions. ${ }^{24}$ In this study, the cell-based experiments revealed that miR-21 protects against IRI and promotes the proliferation of HCC cells by directly inhibiting IL-12A expression. Interleukin-12A (P35) and IL-12B (P40) can form IL-12 dimer and have inflammatory roles in tumor cells. ${ }^{25}$ Previous reports indicated that deletion of IL-12A (P35) altered the inflammation patterns in the liver, subsequently inducing liver fibrosis. ${ }^{26}$ Liver IRI is the major complication in the early phase after hepatectomy due to an over-activated inflammatory response, and, as a response, cells increase their ability to proliferate to protect against this damage. This may be one of the reasons why severe IRI is prone to the recurrence of tumors. ${ }^{27}$

The current study revealed that miR-21 inhibited IL-12A-induced inflammation, and therefore prevented IRI and promoted cell proliferation in HCC cells. This may be one of the mechanisms by which miR-21 acts as a tumor promotor. Additionally, we found that the AA phenotype of miR-21 rs1292037 decreased miR-21 expression and increased IL-12A expression in both cell-based experiments and clinical results. This weakened the tumorigenic function of miR-21 compared to that of AG/GG patients, thus providing a more accurate prognosis.

Study limitations. One is that our findings have not been validated in animal models. Also, multicenter research on the relationship between the miR-21 rs1292037 $A>G$ polymorphism and HCC development should be performed further. The follow-up research on the above limitations makes the focus of our further study.

In conclusion, we found that the AA genotype of miR-21 can down-regulate miR-21 expression in HCC patients, thereby weakening the inhibitory effect of miR-21 on IL-12A. This promotes IRI, but inhibits the proliferation of cancer cells, and can thus predict a more accurate prognosis.

Acknowledgment. The authors gratefully acknowledge Editage (https://wwweditage.com/) for English language editing.

\section{References}

1. Torre LA, Bray F, Siegel RL, Ferlay J, Lortet-Tieulent J, Jemal A. Global cancer statistics, 2012. CA Cancer J Clin 2015; 65: 87-108.

2. Sia D, Villanueva A, Friedman SL, Llovet JM. Liver cancer cell of origin, molecular class, and effects on patient prognosis. Gastroenterology 2017; 152: 745-761.

3. Yao C, Li G, Cai M, Qian Y, Wang L, Xiao L, et al. Expression and genetic polymorphism of necroptosis related protein RIPK1 is correlated with severe hepatic ischemia-reperfusion injury and prognosis after hepatectomy in hepatocellular carcinoma patients. Cancer Biomark 2017; 20: 23-29.

4. Murakami Y, Tanahashi T, Okada R, Toyoda H, Kumada T, Enomoto M, et al. Comparison of hepatocellular carcinoma miRNA expression profiling as evaluated by next generation sequencing and microarray. PLoS One 2014; 9: e106314.

5. Xie Y, Yao Q, Butt AM, Guo J, Tian Z, Bao X, et al. Expression profiling of serum microRNA-101 in HBV-associated chronic hepatitis, liver cirrhosis, and hepatocellular carcinoma. Cancer Biol Ther 2014; 15: 1248-1255. 
6. Moszyńska A, Gebert M, Collawn JF, Bartoszewski R. SNPs in microRNA target sites and their potential role in human disease. Open Biol 2017; 7: 170019.

7. Han BW, Li ZH, Liu SF, Han HB, Dong SJ, Zou HJ, et al. A comprehensive review of microRNA-related polymorphisms in gastric cancer. Genet Mol Res 2016; 15: 1-10.

8. Papaconstantinou I, Kapizioni C, Legaki E, Xourgia E, Karamanolis G, Gklavas A, et al. Association of miR-146 rs2910164, miR-196a rs11614913, miR-221 rs113054794 and miR-224 rs188519172 polymorphisms with anti-TNF treatment response in a Greek population with Crohn's disease. World J Gastrointest Pharmacol Ther 2017; 8: 193-200.

9. Ge Y, Zhang L, Nikolova M, Reva B, Fuchs E. Strand-specific in vivo screen of cancer-associated miRNAs unveils a role for miR-21(*) in SCC progression. Nat Cell Biol 2016; 18 : $111-121$.

10. Ma X, Conklin DJ, Li F, Dai Z, Hua X, Li Y, et al. The oncogenic microRNA miR-21 promotes regulated necrosis in mice. Nat Commun 2015; 6: 7151.

11. Wu C, Wang X, Zhang J, Tang G, Xu Y, Lu C, Li Y. MicroRNA224 expression and polymorphism predict the prognosis of hepatitis B virus-related hepatocellular carcinoma patients after liver resection. Clin Lab 2019; 65.

12. Ding W, Yang H, Gong S, Shi W, Xiao J, Gu J, et al. Candidate miRNAs and pathogenesis investigation for hepatocellular carcinoma based on bioinformatics analysis. Oncol Lett 2017; 13: 3409-3414.

13. Jiang X, Lew KS, Chen Q, Richards AM, Wang P. Human mesenchymal stem cell-derived exosomes reduce ischemia/ reperfusion injury by the inhibitions of apoptosis and autophagy. Curr Pharm Des 2018; 24: 5334-5341.

14. Xu HX, Pan W, Qian JF, Liu F, Dong HQ, Liu QJ. MicroRNA 21 contributes to the puerarin induced cardioprotection via suppression of apoptosis and oxidative stress in a cell model of ischemia/reperfusion injury. Mol Med Rep 2019; 20: 719-727.

15. Zucman-Rossi J, Villanueva A, Nault JC, Llovet JM. Genetic landscape and biomarkers of hepatocellular carcinoma. Gastroenterology 2015; 149: 1226-1239.

16. Mathew S, Abdel-Hafiz H, Raza A, Fatima K, Qadri I. Host nucleotide polymorphism in hepatitis B virus-associated hepatocellular carcinoma. World J Hepatol 2016; 8: 485-498.

17. Petrini E, Caviglia GP, Abate ML, Fagoonee S, Smedile A, Pellicano R. MicroRNAs in HBV-related hepatocellular carcinoma: functions and potential clinical applications. Panminerva Med 2015; 57: 201-209.
18. Li X, Wu X. MiR-21-5p promotes the progression of nonsmall-cell lung cancer by regulating the expression of SMAD7. Onco Targets Ther 2018; 11: 8445-8454.

19. Han Z, Chen Y, Zhang Y, Wei A, Zhou J, Li Q,et al. MiR-21/ PTEN axis promotes skin wound healing by dendritic cells enhancement. J Cell Biochem 2017; 118: 3511-3519.

20. Qiu YF, Wang MX, Meng LN, Zhang R, Wang W. MiR-21 regulates proliferation and apoptosis of oral cancer cells through TNF-a. Eur Rev Med Pharmacol Sci 2018; 22: 7735-7741.

21. Zhuang C, Jiang W, Huang D, Xu L, Yang Q, Zheng L, et al. Serum miR-21, miR-26a and miR-101 as potential biomarkers of hepatocellular carcinoma. Clin Res Hepatol Gastroenterol 2016; 40: 386-396.

22. Huang CS, Yu W, Cui H, Wang YJ, Zhang L, Han F, et al. Increased expression of miR-21 predicts poor prognosis in patients with hepatocellular carcinoma. Int J Clin Exp Pathol 2015; 8: 7234-7238.

23. Yi PS, Li JS. High expression of miR-21 is not a predictor of poor prognosis in all patients with hepatocellular carcinoma. Mol Clin Oncol 2018; 8: 733-739.

24. Wang SM, Zeng WX, Wu WS, Sun LL, Yan D. Association between a microRNA binding site polymorphism in SLCO1A2 and the risk of delayed methotrexate elimination in Chinese children with acute lymphoblastic leukemia. Leuk Res 2018; 65: 61-66.

25. Shi L, Lin H, Li G, Jin RA, Xu J, Sun Y, et al. Targeting androgen receptor $(\mathrm{AR}) \rightarrow$ IL12A signal enhances efficacy of sorafenib plus NK cells immunotherapy to better suppress HCC progression. Mol Cancer Ther 2016; 15: 731-742.

26. Karababa A, Groos-Sahr K, Albrecht U, Keitel V, Shafigullina A, Görg B, Häussinger D. Ammonia Attenuates LPS-Induced Upregulation of Pro-Inflammatory Cytokine mRNA in Co-Cultured Astrocytes and Microglia. Neurochem Res 2017; 42: 737-749.

27. Zhang CY, Yuan WG, He P, Lei JH, Wang CX. Liver fibrosis and hepatic stellate cells: Etiology, pathological hallmarks and therapeutic targets. World J Gastroenterol 2016; 22: 1051210522 . 